\title{
Born-Kothari Condensation for Fermions
}

\author{
Arnab Ghosh ${ }^{\dagger}$ \\ Department of Physical Chemistry, Indian Association for the Cultivation of Science, Jadavpur, Kolkata 700032, \\ India; arnab.ghosh@weizmann.ac.il \\ † Current address: Department of Chemical Physics, Weizmann Institute of Science, 76100 Rehovot, Israel.
}

Received: 12 June 2017; Accepted: 6 September 2017; Published: 13 September 2017

\begin{abstract}
In the spirit of Bose-Einstein condensation, we present a detailed account of the statistical description of the condensation phenomena for a Fermi-Dirac gas following the works of Born and Kothari. For bosons, while the condensed phase below a certain critical temperature, permits macroscopic occupation at the lowest energy single particle state, for fermions, due to Pauli exclusion principle, the condensed phase occurs only in the form of a single occupancy dense modes at the highest energy state. In spite of these rudimentary differences, our recent findings [Ghosh and Ray, 2017] identify the foregoing phenomenon as condensation-like coherence among fermions in an analogous way to Bose-Einstein condensate which is collectively described by a coherent matter wave. To reach the above conclusion, we employ the close relationship between the statistical methods of bosonic and fermionic fields pioneered by Cahill and Glauber. In addition to our previous results, we described in this mini-review that the highest momentum (energy) for individual fermions, prerequisite for the condensation process, can be specified in terms of the natural length and energy scales of the problem. The existence of such condensed phases, which are of obvious significance in the context of elementary particles, have also been scrutinized.
\end{abstract}

Keywords: fermionic coherent states; reciprocity principle; Grassmann algebra

\section{Introduction}

The basic idea of Bose-Einstein condensation (BEC) dates back to 1925 when Einstein [1], on the basis of a work by Bose [2], predicted the occurrence of a phase transition in a gas of noninteracting bosons. The phase transition is the consequence of quantum statistical effects which is associated with the condensation of atoms in the lowest energy state. For a decade these predictions had no practical impact. It was only after the discovery of superfluidity [3] in 1938, the phenomenon received tremendous impetus which eventually led to its experimental realization in 1995 [4-6]. The study of BEC in dilute gases exhibits quantum phenomena on a large scale and has become one of the most active fields of research in atomic, molecular, and condensed matter physics in recent times [7-11].

Interestingly, in the same period around 1938, based on Born's suggestion on "The Theory of Reciprocity Principle" [12,13], the possibility of "condensation" in Fermi-Dirac (FD) statistics [14] was speculated by Kothari and Nath which remained in a dormant state since 1943. Here we review our recent [15] research devoted to elucidate this hitherto unexplored phenomenon of condensation for fermions, in resemblance to BEC. In case of BEC, particles that obey Bose-Einstein (BE) statistics, merge to form a "coherent matter wave" at the lowest energy state. On the other hand, fermions obeying FD statistics, are forbidden to get together by the "Pauli Exclusion Principle" of quantum mechanics. Nonetheless, the above theory can be shown to form a condensate as a dense band at the highest energy state [14] as a direct consequence of Born-Green reciprocity principle which fixes an upper limit of the momentum possible for a particle [16-18]. Motivated by the coherent matter wave description of BEC and a toy model of an ideal gas of non-interacting fermions, we identify the so-called Born-Kothari condensation (BKC) as condensation-like coherence within fermions. While we 
emphasize that the validity of Born-Green reciprocity theory is the hypothesis of our work, it is useful to make some general remarks on the status of reciprocity principle in connection with the current state of affairs:

- The principle of reciprocity originally proposed by Born can be observed in much, but not all, of the formalism of classical and quantum mechanics [19]. However, the idea was later taken up by Yukawa [20] in developing nonlocal quantum field theory [21-23] by recognizing "reciprocity principle as the leading heuristic idea in the theory of elementary particles" [24].

- It is important to point out that the theory of reciprocity is based on a demand for symmetry, requiring the fundamental laws of nature to remain unaltered as mentioned by Born in his letters to Einstein [24]. Although its universality as a general theory remains questionable not only in view of General Relativity which breaks this symmetry [25], but also, there is no extant empirical evidence that could confirm its validity. Current observations in high energy physics experiments suggest that if Born's reciprocity would be valid, then its effect could only be present at energies of the order of $\mathrm{TeV}$ or higher.

- Yet, constructing a general theory defining Born reciprocity as an intrinsic symmetry [26], continued as an interesting field of research even in modern times by adapting group theoretical methods [27-31] which are employed to study a group, namely the quaplectic group. With the advent of renormalization theory and Higg's mechanism in the last seventy years [32-35], both in theory as well as in experiments, it has also been suggested that Born's reciprocity may be the underlying physical reason for the T-duality symmetry in string theory $[25,35,36]$, and may be of relevance in developing a quantum geometry $[37,38]$.

Here we follow the celebrated work of Cahill and Glauber [39] to present a convenient description of the aforementioned condensate for fermions on the basis of a close parallelism between the properties of coherent states for fermionic fields and more familiar ones for bosonic fields. This resulted in an unification between the two seemingly distinct seminal works. Our mini-review is composed of four different kinds of approaches or formulations which are organized as follows: In Section 2, we give an outline of the Born-Green formalism of reciprocity theory to stimulate our discussion, followed by a brief overview in Section 3, on the basic concepts of Kothari-Nath approach regarding the condensation in FD statistics. We summarize in Section 4 , the essential elements of the coherent state formulation for fermionic fields as put forwarded by Cahill and Glauber. Our approach [15] has been discussed in Section 5 and extended further to unravel the BKC as a state of macroscopic condensation-like coherence for fermions within the purview of elementary particles. We conclude our discussion in Sections 6 and 7 and finally, an outlook for open future problems in this direction has been presented in Section 8.

\section{The Principle of Reciprocity: The Born-Green Formalism}

The duality-symmetry among the configuration space and the momentum space was introduced by Born and his collaborator Green to formulate the "principle of reciprocity" [12,13,16-18,21,24,40-43]. According to this, the fundamental laws of nature must be invariant under the linear transformation

$$
x_{k} \longrightarrow p_{k}, \quad p_{k} \longrightarrow-x_{k}, \quad(k=1,2,3,4),
$$

or, more precisely, they are symmetrical in space-time $x^{k} \equiv(x, c t)$ and momentum-energy $p_{k} \equiv$ $(p, E / c)$. The most obvious indications of such invariance under the above transformation are already presented in the accepted laws of classical physics,

$$
\dot{x}^{k}=\partial H / \partial p_{k}, \quad \dot{p}_{k}=-\partial H / \partial x_{k}
$$


when the first 3 components of the 4 -vectors $x^{k}$ and $p_{k}$ are considered. The same symmetry appears in the fundamental commutation relations of relativistic quantum mechanics,

$$
x^{k} p_{l}-p_{l} x^{k}=i \hbar \delta_{l}^{k}
$$

in the Fourier transformation connecting wave functions, and representations of dynamical variables in the co-ordinate and momentum spaces [16], and also in the formal expression for the angular momentum tensor,

$$
j_{k l}=x_{k} p_{l}-x_{l} p_{k}
$$

These observations strongly motivated Born that each general law in the $x$-space has an "inverse image" in the $p$-space [18] and laid him to make an attempt to construct a more general theory which however remained largely unsuccessful.

The most appealing features of reciprocity principle is the introduction of a line element in the $p$-space, $d \sigma^{2}=\gamma^{k l} d p_{k} d p_{l}$, where $\gamma^{k l}$ forms the metric tensor in exact analogy to the coefficients $g_{k l}$ for the four dimensional line element $d s^{2}=g_{k l} d x^{k} d x^{l}$ [12]. The three dimensional volume element in $p$-space is then given by

$$
d \Omega=\frac{d p}{\sqrt{\left(1-p^{2} c^{2} / E^{2}\right)}} .
$$

This volume element comes from the hypotheses that the p-manifold is a closed and maximally symmetric space which is needed in order to derive Lorentz-invariant theory without uv-divergences. The relation between energy $E$ and mass $m$ of a physical system, $E=m c^{2}$, defines the highest momentum $E / c$ corresponding to the rest mass of the particle. One may introduce a similar invariant space-time-momentum-energy line element under quaplectic transformations which assumes Born reciprocity as an accepted group of intrinsic symmetry. If such quaplectic group is considered to be fundamental, then it would imply a maximum rate of change of momentum and appear as a new fundamental constant [26]. Here, however, we confine our discussion in the light of Born-Green reciprocity and its validity is the heart of our analysis. We emphasize that there remains a well distinction between the momentum and the coordinate space, because the 4-dimensional distance $R^{\frac{1}{2}}$, for the latter, with $R=x^{k} x_{k}=c^{2} t^{2}-x^{2}$, has an absolute significance and can be measured for macroscopic dimensions. Natural question therefore arises, is it plausible to measure 4-dimensional distance between two events in microscopic dimensions too?

From the standpoint of momenta, there exists of course a quantity analogous to $R$, namely $P=p_{k} p^{k}=E^{2} / c^{2}-p^{2}$. However, this is not a continuous variable since it involves the rest mass. A determination of $P$, therefore implies not a real measurement but a choice between a number of values corresponding to the particles. By estimating $p$ and $E$ for a particle, one obtains the value of the rest mass which permits to recognize the kind of particles with which one is dealing with [18].

We conclude this section with a note that the idea of "reciprocity" is a generalization of the invariant "metric-operator" concept as an extension of the Minkowski metric of special relativity. The simplest relativistically and reciprocally invariant metric function constructed by Born and Green, comprises the $x$-space and $p$-space simultaneously [16], i.e.,

$$
S=x_{k} x^{k}+p_{k} p^{k}
$$

For further generalization see References [25,34-36,44,45]. Using the notations $R=x_{k} x^{k}$ and $P=p_{k} p^{k}$, introduced above, Equation (6) can be rewritten as

$$
S=R+P .
$$


The metric invariant $R$, which is a number scalar in $x$-space, is replaced by Equation (7) as the symmetric sum of the $x$ and $p$-space metric operators. As long as we are dealing with macroscopic bodies in macroscopic dimensions, $P$ is small and in all such situations ordinary relativity holds as a perfect assumption. However, on a microscopic scale, the space metric is not independent of the momentum metric, both together determine $S$. It may be worthwhile to clarify a small ambiguity at this point: According to the reciprocity principle, Einstein special relativity is an accurate theory for macroscopic domain [18], but this does not imply that one should expect its effect in all every day experiments. It is well known that at small velocities relative to the speed of light, Newtonian mechanics is completely an accurate description for macroscopic world. Similarly, in atomic physics where one basically deals with a $1 / r$ potential, Born reciprocity has negligible effect on known experimental results [46]. It therefore implies that the standard relativistic tools are perfectly adequate methods for known experimental scenarios. The same is true in the context of a condensed matter problem. Interestingly enough, this point was nicely demonstrated with several examples (including the deviation from standard Planck's distribution law) by Born that the correction due to reciprocity principle (although very small) makes sense only at extremely high energy (or equivalent temperature) [12]. An estimation of the aforesaid energy scale following Born is given by $k_{B} \Theta_{0} \gg 1.41 \times 10^{8} \mathrm{eV}$, which in terms of temperature corresponds to $\Theta_{0} \gg 1.63 \times 10^{12}$ degrees (See Equation (37) of Reference [12]). Since in the last twenty years there are experimental data reporting that electrons may reach $200 \mathrm{GeV}$ [47], it suggests that any modification in the density of states due to reciprocity principle may only appear at energies $\gtrsim 1 \mathrm{TeV}$ (see also Discussion Section 6).

Finally, as $x$ and $p$ have different dimensions, it is customary to measure all coordinates $x^{k}$ in units $a$ and all $p_{k}$ in units $b$, so that they become dimensionless. Instead of $a$ and $b$ one may choose Planck's constant as the primary absolute constant $[18,40]$, so that

$$
\hbar=a \cdot b \text {. }
$$

The actual mass $\mu$ of an elementary particle can then be computed from its reduced rest mass $\kappa$, using the formula $\mu=\hbar \kappa / a c$ (Appendix A), where $a$ is the classical electron radius, the natural length scale of electron and simultaneously of all nuclei. Finally, we point out that the highest momentum of any particle is related to its reduced rest mass $\kappa$, by the relation

$$
\frac{E}{c}=\mu \cdot c=\kappa \cdot b .
$$

Equation (9) which is our new observation of the present analysis, reflects an interesting point. It states that the highest momentum of a particle is specific to its rest mass and the product of the reduced rest mass and the scale factor $b$. We will use this relation in the next section and return to its discussion in Section 6 , on the choice of the scale parameter $b$ to account for the characteristic Born constant of the reciprocity theory. Once again we stress that the Born-Green reciprocity theory is the hypothesis of our work and our analysis based on a toy model of an ideal gas of non-interacting fermions, entirely depends on the validity of the above principle.

\section{3. "Condensation" in FD Statistics: The Kothari-Nath Approach}

Theory of Reciprocity, thus developed by Born and Green, plays the role for the momentum coordinates what Einstein's theory does for position coordinates. Following Equations (5) and (9), and making use of the hypothesis connecting the proportionality relation between the density of states and the volume element, the number of wave-functions for a particle with weight factor $g$ (due to its internal degrees of freedom), enclosed in a volume $V$ and momentum range $p$ to $p+d p$ is given by

$$
a(p) d p=\frac{4 \pi V g}{(2 \pi \hbar)^{3}} \frac{p^{2} d p}{\left(1-p^{2} / \kappa^{2} b^{2}\right)^{1 / 2}} .
$$


At this point, let us take a pause and make some important upshot in view of Equation (10):

- It is apparent from Equation (10) that the only effect of the reciprocity principle is on the density of states. Importantly, it was pointed out by Born, "a consequence of this assumption is a modification of the formula for the number of quantum states" [12].

- In the usual treatment the momentum space is taken to be infinite, i.e., $b \rightarrow \infty$ [48]. Then Equation (10) reduces to the well known expression $a(p) d p=\frac{4 \pi V g}{(2 \pi \hbar)^{3}} p^{2} d p$ [49].

- The form of the statistical distributions, either, Fermi-Dirac, Bose-Einstein, or Maxwell-Boltzmann, remain unperturbed by the reciprocity principle [12,48].

It is of interest, therefore, to see to what extent the properties of an assembly of identical and non-interacting particles get modified when the momentum space presumed by Born is restricted in the above manner. While the case of a classical gas and that of a radiation field have been analysed by Born himself [12], the thermodynamic properties of both relativistic and non-relativistic FD gas have been studied by others [48]. Remarkably, all the thermodynamic relations are found to be exactly identical to their well known results.

However, the most intriguing consequence of the reciprocity principle was due to Kothari and Nath [14] who pointed out the possible existence of a "condensed phase" in FD statistics. Unlike Bose gases, where the condensed phases are formed by the particles in the lowest energy state, for FD gases, the condensed phases are formed by the particles in their respective highest energy states. According to Equation (10), the total number of wave-functions $\left(a_{0}\right)$ contained in a volume $V$ is

$$
a_{0}=\int_{0}^{\kappa b} a(p) d p=g \frac{\pi^{2} V \kappa^{3} b^{3}}{(2 \pi \hbar)^{3}}
$$

which can also be expressed in terms of the Compton's wavelength $\lambda_{c}=\frac{h}{\mu c}$ of the particle (i.e., the wavelength of a photon whose energy is equal to the mass of the particle) as $a_{0} \propto g V / \lambda_{c}^{3}$. Equation (11) determines the maximum number of possible wave-functions $\left(N \equiv N_{0}=a_{0}\right)$ that can be accommodated by the volume $V$. Again by Pauli exclusion principle, any independent wave-function for fermions can only be occupied by a single particle. It is, therefore, a legitimate question to ask what will happen to the FD gas, when the number of particles $N$ in the volume $V$, exceeds $N_{0}$ ?

Kothari and Nath [14] resolved the issue by proposing that on replacement of the sum by the integral of all possible eigenstates extending from $(p=0)$ to $(p=E / c)$ in Equation (11), the total number of eigenstates although becomes limited, it is practically infinite. So, the total number of states a, can be written as

$$
\mathbf{a}=a_{0}+a_{1}
$$

where $a_{1}$ denotes an infinitely large number of eigen-states corresponding to the highest momentum $p \rightarrow \kappa b$, which has a specific value for a fixed fermion rest mass. It follows, therefore when the number of particles $N$ in a FD gas exceed $N_{0}$, irrespective of any critical temperature, a condensed phase containing $\left(N-N_{0}\right)$ particles, will be formed in the highest energy state, all possessing momentum $E / c$. One may expect that the existence of such "condensed" phases are of natural significance for the application of the reciprocity theory within the ambit of elementary particles.

Here we draw the attention of the readers to the crucial step behind Kothari-Nath proposition. It is generally assumed that the density of states is proportional to the volume element in the $p$-space as we considered here in Equation (10). This is true in the description of BEC which is derived by assuming a discrete set of plane wave modes in a large box, and then allowing the box length to infinity to recover the integral as a limit of the sum. Here on the other hand, Born reciprocity in the form of Equation (10) does not establish which are the modes; it just specifies the volume element in the $p$-space. This difference is the key point in the argument of Kothari and Nath, where they presume 
that the integral in Equation (11) comes from a sum. Moreover, the ad-hoc term $a_{1}$ in Equation (12) is included to force the system to incorporate an infinite number of particles in a fixed volume.

One should keep in mind that the above inference is not in contradiction with our basic knowledge that asserts fermions obey Pauli exclusion principle, ruling out the possibility of macroscopic occupation at a single particle state [50]. Yet, to this end, we pose the following question: Is it still possible to draw a close parallelism between the condensation of Reference [14] and that of BEC? Using the statistical properties for fermionic fields in kinship to bosonic fields, pioneered by Cahill and Glauber [39], we have shown in the following that the desired concordance can be arrived within the framework of coherent states. The prime advantage of such coherent state formalism is that it is no more regulated by the strict Yang criterion [50] for fermionic fields that must abide by the single particle picture. This accounts for a consistent and satisfactory theory for the largely untrodden condensation event of the FD gas in terms of fermionic coherent states. In what follows, we first make a little digression on the coherent states of fermions followed by our methods that conceives BKC as a close BEC analog.

\section{Fermionic Coherent States: The Cahill-Glauber Formulation}

The mathematical methods that are used to characterize the statistical properties of boson fields, in particular the coherence of photons in quantum optics, has their counterparts for fermionic fields. The coherent states, displacement operators, $P$-representation all possess surprisingly close fermionic analogues. However, an extension of these schemes to their fermionic counterpart is not a trivial task. The reason was first pointed out by Schwinger [51], since fermion field variables anticommute, their eigenvalues must be anticommuting numbers. Such numbers are called Grassmann numbers which can be handled only by means of Grassmann algebra. For general properties of such anti-commuting Grassmann variables we refer the reader to the classic book of Berezin [52]. Here instead we follow the line of approach publicized by Cahill and Glauber [39] for the construction of fermionic coherent states to describe the statistical properties of fermionic fields. In doing so, we summarize only the parts necessary to make our presentation self-contained.

However, one can proceed one step further and ask in the light of the extended form of relativity, due to Born, what will happen with locality and micro-causality in the context of relativistic and eventually in reciprocal quantum field theories? Since coordinate space is curved while the momentum space is linear and therefore flat, it appears that the introduction of gravity breaks the symmetry between them. Still there are literatures which considered curved momentum space geometry to allow a natural way to reconcile gravity with quantum mechanics and discuss relative locality [53] and micro-causality $[34,54]$ from a newer perspective $[35,55]$. In this connection we mention earlier works relating reciprocity with non-localizable quantum fields by Born [21], Pias [22] and Yukawa [20] which made a significant contribution to the development of meson theories in 50's. We stress however that this is of course not relevant for our present purpose, since we restrict our discussion within non-interacting fermions. Therefore, we refrain ourselves further in this direction as it is beyond the scope of our present discussion.

\subsection{Properties of Anti-Commuting Numbers}

For a system of fermions described by the creation $a_{k}^{\dagger}$ and annihilation $a_{l}$ operators following

$$
\left\{\hat{a}_{k}, \hat{a}_{l}^{\dagger}\right\}=\delta_{k l} ; \quad\left\{\hat{a}_{k}, \hat{a}_{l}\right\}=\left\{\hat{a}_{k}^{\dagger}, \hat{a}_{l}^{\dagger}\right\}=0,
$$

the set of Grassmann variables $\gamma=\left\{\gamma_{k}\right\}$ satisfy the anti-commutation relations,

$$
\gamma_{k} \gamma_{l}+\gamma_{l} \gamma_{k} \equiv\left\{\gamma_{k}, \gamma_{l}\right\}=0
$$

This immediately results in, for any given " $k$ ", $\gamma_{k}^{2}=0$. As the square of every Grassmann monomial vanishes, any Grassmann variable can not be an ordinary real, imaginary, or complex number. In other words, they are nilpotent, an important property for the treatment of fermions [52,56-58]. This 
crucial difference between the Grassmann variables and the ordinary variables has far-reaching consequences, such as, the operation of differentiation is the same as the integration in Grassmann calculus. The Grassmann number $\gamma_{k}$ and its complex conjugate $\gamma_{k}^{*}$ are independent variables which obey

$$
\left\{\gamma_{k}, \gamma_{k}^{*}\right\}=0
$$

They also anti-commute with their fermionic operators, like:

$$
\left\{\gamma_{k}, \hat{a}_{l}\right\}=0 ; \quad\left\{\gamma_{k}, \hat{a}_{l}^{\dagger}\right\}=0
$$

The Hermitian conjugation reverses the order of all fermionic quantities, both the operators and the anti-commuting numbers. For an example

$$
\left(\hat{a}_{1} \gamma_{2} \hat{a}_{3}^{\dagger} \gamma_{4}^{*}\right)^{\dagger}=\gamma_{4} \hat{a}_{3} \gamma_{2}^{*} \hat{a}_{1}^{\dagger}
$$

While Grassmann numbers are well studied in mathematics [52] and path integral formulation for fermionic fields [56-58], other applications of such anti-commuting numbers are less popular. Recently, however, a number of investigations by incorporating Grassmann variables in fermionic systems have appeared in the literature [59-76]. Among them are the phase space methods for degenerate Fermi gases [59], counting statistics and quantum Monte-Carlo methods of strongly correlated fermions [62], superfluidity [67] and Cooper-like pairing in trapped fermions [68], to name just a few.

\subsection{Coherent States for Fermions}

The entire formulation of quantum optics is couched in terms of coherent states [77-80], which are eigen-states of the harmonic oscillator annihilation operators and obey bosonic commutation relations. For fermionic fields, though, the vacuum state is the only physically realizable eigenstate of the annihilation operators, still, it is possible, to define such eigenstates in a formal way and put them to the same analytical uses as they are made for bosonic coherent states.

Thus, in analogy to coherent state $|\boldsymbol{\alpha}\rangle$ [77-80] for bosonic field which is a displaced state $|\boldsymbol{\alpha}\rangle=$ $\hat{\mathbf{D}}(\boldsymbol{\alpha})|0\rangle$ produced by the action of the displacement operator $\hat{\mathbf{D}}(\boldsymbol{\alpha})=\exp \left(\sum_{i}\left(\alpha_{i} \hat{a}_{i}^{\dagger}-\alpha_{i}^{*} \hat{a}_{i}\right)\right)$ on the vacuum $|0\rangle$, where $\left\{\alpha_{i}\right\}$ being a set of complex numbers [77-84], it is possible to construct the normalized coherent state for fermions [39].

$$
|\gamma\rangle=\hat{\mathcal{D}}(\gamma)|0\rangle
$$

Here the displacement operator is given by

$$
\hat{\mathcal{D}}(\gamma)=\exp \left(\sum_{i}\left(\hat{a}_{i}^{\dagger} \gamma_{i}-\gamma_{i}^{*} \hat{a}_{i}\right)\right),
$$

for a set of $\gamma=\left\{\gamma_{i}\right\}$ Grassmann variables. It should be noted that in Equation (19) the creation operator $\hat{a}_{i}^{\dagger}$ must stand to the left of the Grassmann variable $\gamma_{i}$. Using the properties of the displaced annihilation operator

$$
\hat{\mathcal{D}}^{\dagger}(\gamma) \hat{a}_{k} \hat{\mathcal{D}}(\gamma)=\hat{a}_{k}+\gamma_{k}
$$

one can show that the coherent state is an eigenstate of every annihilation operator $\hat{a}_{k}, \hat{a}_{k}|\gamma\rangle=\gamma_{k}|\gamma\rangle$, with eigenvalue $\gamma_{k}$ (see Appendix B). While the adjoint relation is $\langle\gamma| \hat{a}_{k}^{+}=\langle\gamma| \gamma_{k}^{*}$, the inner product of any two coherent states is given by

$$
\langle\gamma \mid \boldsymbol{\beta}\rangle=\exp \left(\sum_{i} \gamma_{i}^{*} \beta_{i}-\frac{1}{2}\left(\gamma_{i}^{*} \gamma_{i}+\beta_{i}^{*} \beta_{i}\right)\right) .
$$


Upon using the completeness relation of the coherent states, an arbitrary coherent state $|\boldsymbol{\beta}\rangle$ can be expanded by

$$
|\beta\rangle=\int d^{2} \gamma\langle\gamma \mid \beta\rangle|\gamma\rangle
$$

which immediately follows from the resolution of identity

$$
\int d^{2} \gamma|\gamma\rangle\langle\gamma|=\mathbf{I}
$$

Here we emphasize that for fermionic fields the integrations are taken over anticommuting variables and for such pairs we will adhere to the notation $\int d^{2} \gamma \equiv \int \prod_{i} d^{2} \gamma_{i}, \int d^{2} \gamma_{i}=\int d \gamma_{i}^{*} d \gamma_{i}$, in which the differential of the conjugate variable comes first and we keep in mind that $d \gamma_{i}^{*} d \gamma_{i}=-d \gamma_{i} d \gamma_{i}^{*}$.

Now, the most conspicuous feature for fermionic field arises from the peculiar property of fermionic oscillator which has both upper and lower bounds [57], in contrast to only lower bound for harmonic oscillator in case of a bosonic field. This ensures unlike bosonic fields, it is possible to define the normalized eigenstate $|\boldsymbol{\alpha}\rangle^{\prime}$ of the fermionic creation operators $\hat{a}_{k}^{\dagger}$ for any set $\alpha=\left\{\alpha_{i}\right\}$ of Grassmann variables, as the displaced state

$$
|\boldsymbol{\alpha}\rangle^{\prime}=\hat{\mathcal{D}}(\boldsymbol{\alpha})|\mathbf{1}\rangle,
$$

where $|\mathbf{1}\rangle$ represents the state in which every mode is filled:

$$
|\mathbf{1}\rangle=\prod_{k} \hat{a}_{k}^{\dagger}|0\rangle .
$$

Using the adjoint relation of Equation (20) (confer Equation (A10)), one can show that the state $|\boldsymbol{\alpha}\rangle^{\prime}$ is an eigenstate of every creation operator $\hat{a}_{k}^{\dagger}$ (see Appendix B):

$$
\hat{a}_{k}^{\dagger}|\boldsymbol{\alpha}\rangle^{\prime}=\alpha_{k}^{*}|\boldsymbol{\alpha}\rangle^{\prime} .
$$

Similarly, the corresponding adjoint equation and the identity operator for the eigenstates $|\alpha\rangle^{\prime}$ are given by

$$
\begin{array}{r}
{ }^{\langle\alpha} \mid \hat{a}_{k}={ }^{\prime}\langle\boldsymbol{\alpha}| \alpha_{k} \\
\int \prod_{i}\left(-d^{2} \alpha_{i}\right)|\boldsymbol{\alpha}\rangle^{\prime}{ }^{\prime}\langle\boldsymbol{\alpha}|=\mathbf{I} .
\end{array}
$$

We close this section with the following note:

- As pointed out after Equation (19) that for fermionic field, every operator and Grassmann numbers anticommute with each other. Hence, special care must be taken for the ordering of all such fermionic quantities.

- Eigenstate of every fermionic creation operators, $|\boldsymbol{\alpha}\rangle^{\prime}$, stems from the characteristic upper bound exhibited by general system of fermions. Equation (24) will thus be shown to play the key role in our theoretical formulation which we explore in the next section.

Apart from this ordering issue and the characteristic upper bound possessed by fermionic field modes, Equations (18)-(27) are rather similar to their bosonic counterparts. So, they can be applied to the same analytical techniques as their counterparts are employed for bosonic fields [15,39,72,74,75,85]. The differences prevail only in their mathematical background [52,56-58]. 


\section{BKC as Condensation-Like Coherence within Fermions}

We proceed by considering the Hamiltonian [15]

$$
\hat{\mathcal{H}}=\int\left(\frac{\hbar^{2}}{2 \mu} \nabla \hat{\psi}^{\dagger}(x) \nabla \hat{\psi}(x)\right) d x
$$

of a non-interacting Fermi gas of $N$ particles of mass $\mu$, expressed in terms of the field operators $\hat{\psi}(x)$ and $\hat{\psi}^{\dagger}(x)$. The field operator $\hat{\psi}(x)$ that annihilates a particle at a space point $x$ can be expanded in terms of its mode functions $\phi_{k}(x)$ as

$$
\hat{\psi}(x)=\sum_{k} \hat{a}_{k} \phi_{k}(x)
$$

The field operator $\hat{\psi}(x)$ and its adjoint satisfy the anti-commutation relation

$$
\left\{\hat{\psi}(x), \hat{\psi}^{\dagger}\left(x^{\prime}\right)\right\}=\sum_{k} \phi_{k}(x) \phi_{k}^{*}\left(x^{\prime}\right)=\delta\left(x-x^{\prime}\right),
$$

which implies that the creation $\left(\hat{a}_{k}^{+}\right)$and annihilation $\left(\hat{a}_{k}\right)$ operators in the single particle state $\phi_{k}(x)$ obey the fermionic anti-commutation relations Equations (13), while wave functions $\phi_{k}(x)$ fulfil the orthonormal condition

$$
\int \phi_{k}^{*}(x) \phi_{l}(x)=\delta_{k l}
$$

The close similarity between the bosonic and fermionic fields [39] discussed in the previous section and a hint from Bogoliubov approximation [86] for bosons motivate us to separate out the field operator $\hat{\psi}(x)$ for a systematic description into a non-commuting "classical" field $\psi(x)$ and the quantum fluctuation $\delta \hat{\psi}(x)$ about its classical component,

$$
\hat{\psi}(x)=\psi(x)+\delta \hat{\psi}(x) .
$$

The sense of "classicality" for fermionic fields will be addressed later on, in course of examining fermionic "order parameter". As evident, the ansatz for the Equations (32) is guided by the non-zero expectation value $\langle\hat{\psi}(x)\rangle$ for the harmonic oscillator coherent states for bosonic fields which are well-known basis for examining the condensation phenomena in BEC [7-9]. In the same spirit we will utilize fermionic oscillator coherent states introduced by Cahill and Glauber [39] to explain the condensation behaviour of the FD gas envisaged by Kothari and Nath [14] on the basis of Born-Green reciprocity theory $[17,18]$.

\subsection{Comparative Study between BEC and BKC}

In the following we first lay down the steps behind our preliminary surmise to depict BKC in analogy to BEC:

1. In BEC, the condensed phase forms a coherent matter wave as the ground state of the many body system. Hence it is quite logical to delineate the giant matter wave in terms of a bosonic coherent state which is a displaced vacuum state $|\alpha\rangle=\hat{\mathbf{D}}(\boldsymbol{\alpha})|0\rangle$, with, $\alpha$ being the complex numbers (Figure 1a).

2. On the other hand, as discussed in Section 2, the condensed phase for a FD gas occurs only at the highest energy state $p=\kappa b$, characteristic of the reduced mass of the fermionic system. The reduced mass (rest mass) in case of BKC is analogous to $T_{\mathcal{C}}$ for bosonic systems that dictates the existence of BEC below such critical temperatures. 
3. Due to Pauli exclusion principle, any eigenstate of fermions can accommodate only a single particle. Subsequently, by reciprocity principle the highest momentum state $p=\kappa b$ for any fermionic system, in principle contains an infinitely large number of eigenstates [14].

4. Combining the points 2 and 3 , unequivocally suggest that the condensed phase for each individual fermionic systems can occur only in the form of a single occupancy dense band at their respective highest accessible momentum (energy) state, $p=\kappa b$ (Figure 1b).

5. Since, the eigenstate of the fermionic creation operator originates from the characteristic upper bound displayed by any general systems of fermions, our preliminary surmise is that the condensate of Reference [14] that appears at the highest energy state in the form of a dense band, is most appropriately described in light of our Equation (24), as a displaced state $|\boldsymbol{\alpha}\rangle^{\prime}=\hat{\mathcal{D}}(\boldsymbol{\alpha})|\mathbf{1}\rangle$. As discussed in Section 3, that the Born reciprocity does not specify the modes, so an explanation of the exclusion principle focusing on the quantum numbers for the ad-hoc $a_{1}$ term of Equation (12), is not apparent. Therefore we resort to this end to fermionic coherent state in order to establish that the condensed phase at the highest energy is compatible with the exclusion principle. Since fermionic operator anticommutes, any creation operator can act only once on the vacuum, $a_{n}^{\dagger}|0\rangle$, thereby it creates only a single particle in each mode. As a result, $|\mathbf{1}\rangle=\prod_{n=N_{0}}^{n=N} a_{n}^{\dagger}|0\rangle$ which represents product state of all such large number of modes created by the single action of their creation operators, absolutely fulfils the exclusion principle. Thus, a unitary transformation of such state, here for instance, the displaced coherent state $|\boldsymbol{\alpha}\rangle^{\prime}=\hat{\mathcal{D}}(\boldsymbol{\alpha})|\mathbf{1}\rangle$ in Equation (24), automatically obeys the Pauli exclusion principle.

(a)

coherent matter wave

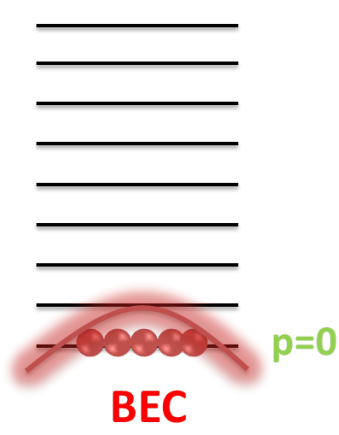

(b)

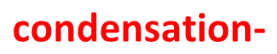
like coherence

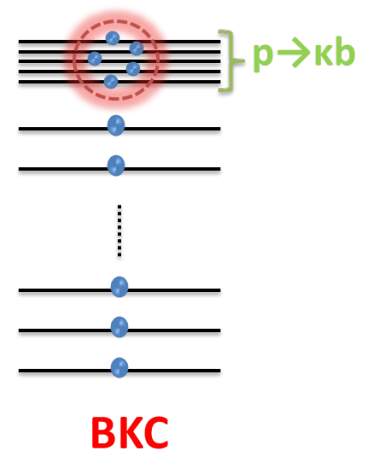

Figure 1. Schematic diagrams illustrating the basic differences and innate similarities between two kinds of condensations: (a) Bose-Einstein condensation (forms coherent matter wave) and (b) Born-Kothari condensation (forms condensation-like coherence).

This allows us to associate an intrinsic notion of symmetry breaking and thermodynamic "order parameter" to characterize the condensation for a gas of non-interacting fermions in parallel to Bose condensate for a gas of non-interacting bosons.

\subsection{Characterization of the "Condensate" State}

The basis for characterization of the "condensed" state of BKC is the thermodynamic limit, order parameter and spontaneous symmetry breaking, as detailed below. 


\subsubsection{Thermodynamic Limit of Fermionic Operators}

We are interested in the behaviour of a gas of fermionic atoms in the thermodynamic limit. Therefore, it is necessary to comprehend both the number of particles and the volume of the gas are large, i.e., $N \longrightarrow \infty$ and $V \longrightarrow \infty$, keeping the density $\rho=\frac{N}{V}$ constant. In this limit, we are allowed to forget the operator character of $\hat{a}_{n}$ and $\hat{a}_{n}^{+}$because

$$
\frac{\left\{\hat{a}_{n}, \hat{a}_{n}^{\dagger}\right\}}{V}=\frac{\hat{a}_{n} \hat{a}_{n}^{\dagger}+\hat{a}_{n}^{\dagger} \hat{a}_{n}}{V}=\frac{1}{V} \longrightarrow 0 .
$$

This is similar to the commutation relations for bosonic operators which vanish in the thermodynamic limit, and thus are replaced by c-numbers in BEC theory $[8,9,49]$. This can be illustrated by considering the non-interacting ground state of a Bose gas with $N$ particles

$$
\left|\Phi_{0}(N)\right\rangle=|N, 0,0,0 \ldots\rangle,
$$

where all $N$ particles occupy the state $k=0$. The annihilation and creation operators for the state $k=0$ are denoted by $\hat{a}_{0}$ and $\hat{a}_{0}^{\dagger}$ which satisfy

$$
\begin{array}{r}
\hat{a}_{0}\left|\Phi_{0}(N)\right\rangle=N^{1 / 2}\left|\Phi_{0}(N-1)\right\rangle, \\
\hat{a}_{0}^{\dagger}\left|\Phi_{0}(N)\right\rangle=(N+1)^{1 / 2}\left|\Phi_{0}(N+1)\right\rangle .
\end{array}
$$

It is important to note that neither $\hat{a}_{0}^{\dagger}$ nor $\hat{a}_{0}$ annihilate the ground state. This is in contrast to the well-known property of $\hat{a}_{0}|0\rangle=0$ of a vacuum state. As the bosonic operators multiply the ground state with large numbers of the order $\sqrt{N}$, it is convenient to switch to new operators defined as

$$
\hat{b}_{0}=V^{-1 / 2} \hat{a}_{0} \quad \text { and } \quad \hat{b}_{0}^{+}=V^{-1 / 2} \hat{a}_{0}^{\dagger} .
$$

Since $\left[\hat{a}_{0}, \hat{a}_{0}^{\dagger}\right]=1$, we get $\left[\hat{b}_{0}, \hat{b}_{0}^{\dagger}\right]=V^{-1}$. Therefore one has

$$
\begin{array}{r}
\hat{b}_{0}\left|\Phi_{0}(N)\right\rangle=\left(\frac{N}{V}\right)^{1 / 2}\left|\Phi_{0}(N-1)\right\rangle, \\
\hat{b}_{0}^{\dagger}\left|\Phi_{0}(N)\right\rangle=\left(\frac{N+1}{V}\right)^{1 / 2}\left|\Phi_{0}(N+1)\right\rangle .
\end{array}
$$

As mentioned before we are interested in the behaviour of a gas in the thermodynamic limit, hence the operators $\hat{b}_{0}$ and $\hat{b}_{0}^{+}$multiply the ground state with a constant factor. Therefore, the commutator

$$
\frac{\left[\hat{a}_{0}, \hat{a}_{0}^{\dagger}\right]}{V}=\frac{\hat{a}_{0} \hat{a}_{0}^{\dagger}-\hat{a}_{0}^{\dagger} \hat{a}_{0}}{V}=\frac{1}{V} \longrightarrow 0,
$$

in the thermodynamic limit, i.e., we obtain a classical limit for which the bosonic operators are replaced by ordinary c-numbers.

In the same way, anticommuting fermionic operators $\hat{a}_{n}$ and $\hat{a}_{n}^{\dagger}$ can be replaced by numbers in the thermodynamic limit, $V \longrightarrow \infty$. The only difference is that unlike the bosonic case where the operators are replaced by classical commuting variables, these numbers must be Grassmann or anti-commuting classical numbers for fermionic case. One can make this point more explicit by defining

$$
\hat{a}_{n}^{\dagger} \hat{a}_{n}=\hat{N}_{n}
$$

Because of ${ }^{\prime}\left\langle\boldsymbol{\alpha}\left|\hat{N}_{n}\right| \boldsymbol{\alpha}\right\rangle^{\prime}=\alpha_{n}^{*} \alpha_{n} \neq 0$, neither $\hat{a}_{n}$ and $\hat{a}_{n}^{\dagger}$ can annihilate the ground state of a system of $N$ fermionic atoms, realized as the coherent state $|\alpha\rangle^{\prime}$. In the thermodynamic limit, we identify $\sum_{n} \alpha_{n}^{*} \alpha_{n}$ as the average number of fermions in the condensed phase. We thus obtain the classical limit of the 
fermionic operators for which $\hat{a}_{n}$ and $\hat{a}_{n}^{+}$are replaced by their corresponding Grassmann variables, which follows from their anti-commuting nature:

$$
\frac{\left\langle\boldsymbol{\alpha}\left|\left\{\hat{a}_{n}, \hat{a}_{n}^{\dagger}\right\}\right| \boldsymbol{\alpha}\right\rangle^{\prime}}{V}=\frac{1+\left\{\alpha_{n}, \alpha_{n}^{*}\right\}}{V}=\frac{1}{V} \longrightarrow 0
$$

In the following we further illustrate the physical significance of these Grassmann variables from the fermionic order parameter point of view.

\subsubsection{Fermionic Order Parameter}

In general, when the quantum fluctuation is neglected, the field operator $\hat{\psi}(x)$ coincides with the classical field $\psi(x)$. This happens for a dilute Bose gas at very low temperature and the system behaves as a classical object [7-9]. For bosonic fields, the function $\psi(x)=\sqrt{N / V}$ plays the role of an order parameter which helps to characterize the basic principle of BEC. Similarly, the fermionic field operator $\hat{\psi}(x)$ expanded in terms of its mode functions $\phi_{k}(x)$ (Equation (29)), in a properly parametrized coherent states

$$
|\boldsymbol{\alpha}\rangle^{\prime}=\exp \left(\sqrt{V} \sum_{n}\left(a_{n}^{\dagger} \alpha_{n}-\alpha_{n}^{*} a_{n}\right)\right)|\mathbf{1}\rangle,
$$

satisfies the eigenvalue equation

$$
{ }^{\prime}\langle\boldsymbol{\alpha}| \hat{\psi}(x){ }^{\prime}\langle\boldsymbol{\alpha}| \psi(x) .
$$

Here the eigenvalue $\psi(x)$ corresponds to the amplitude

$$
\psi(x)=\sum_{n} \alpha_{n} \phi_{n}(x)
$$

of the fermionic or Grassmann field wherein the annihilation operators of $\hat{\psi}(x)$ are replaced by the Grassmann variables $\alpha=\left\{\alpha_{n}\right\}$. As the parametrized coherent states are defined in terms of bilinear forms in anticommuting variables, there is no need to adopt any ordering for the modes or extra minus sign to compute the density of the FD gas, $\rho=N / V$, where $N=\int \psi^{*}(r) \psi(r) d r$. Therefore, like the bosonic order parameter, one can always multiply $\psi(x)$ by a numerical phase factor as $e^{i \theta}$ or equivalently can multiply $\alpha_{n}$ by $e^{i \theta}$, without changing any physical property. This demonstrates the natural gauge symmetry exhibited by all the physical equations of the fermionic fields. Hence, making an explicit choice for the value of the phase, in turn order parameter, corresponds to a formal breaking of the gauge symmetry which is guaranteed to be the necessary and sufficient condition $[87,88]$ for the occurrence of BEC in bosonic fields. We will reveal its significance to their fermionic counterpart in due course, but, before that, several remarks are in order:

- One can argue that the fermionic order parameter does not bear any classical analogy, because it involves Grassmann variables. This may lead to misconception, hence needs further clarification. To be classically measurable, a field amplitude has to be large enough, like that of a strong electromagnetic (EM) field which can be produced and measured classically. This is possible when the particles obey BE statistics, as happens for light quanta in EM fields, where a large number of particles can accommodate a same state so that the fields get summed up coherently. On the other hand, for fermionic fields obeying FD statistics, quantities like energy, charge, current density which are bilinear in field variables, are only physically relevant entities. The reason is the number $\hat{N}=\sum_{k} \hat{a}_{k}^{\dagger} \hat{a}_{k}$ and energy operators $\hat{H}=\sum_{k} \epsilon_{k} \hat{a}_{k}^{\dagger} \hat{a}_{k}$ do have classical limits even for fermionic fields, as they are bilinear in $\hat{a}_{k}$ and $\hat{a}_{k}^{\dagger}$. Extrapolating this idea, we may say that Grassmann fields and fermionic field operators are by nature fermionic, while an even product of these variables make them experimentally relevant $[15,68,70,72,74,75]$. A representation of correlation functions and construction of parent quadratic Hamiltonians in terms of such Grassmann variables can 
be found in recent review $[59,60]$ and in the theory of fermionic analog of parametric amplifier developed by the present author $[74,75]$.

- Therefore the order parameter $\psi(x)$ which is linear in Grassmann variables (Equation (43)), represents the amplitude of the fermionic field and is not an experimentally viable quantity. On the contrary, the field intensity $\psi^{*}(r) \psi(r)=\sum_{n} \alpha_{n}^{*} \alpha_{n}\left|\phi_{n}(r)\right|^{2}$, is bilinear in Grassmann amplitudes, is an experimentally relevant quantity without any ambiguity [15]. Anticommutation in quantum mechanics is very special because it involves Pauli exclusion principle, which does not make sense at the classical level.

- Although the anticommuting nature of Grassmann variables preclude the possibility of interpreting them in experimental terms, we have shown [75] that unlike the bosonic field, fermionic fields are bound to satisfy fermionic analog of classical Liouville's equation [61] and therefore most closely resemble to classical phase-space distribution functions. This is not surprising because of the simple fact that Grassmann algebra does not allow any derivative higher than second order. In this sense, the fermionic field operators being linear in their field variables, are always classical except for the effect that they incorporate Pauli exclusion principle. This is an important difference when compared with their bosonic counterparts since for the latter the Liouville's equation is obtained only when the fields are either free or the quantum correction to the interaction are neglected [75].

\subsubsection{Spontaneous Symmetry Breaking}

Finally, we choose the appropriately parametrized coherent state as Equation (41). We start by noting that the state $|\boldsymbol{\alpha}\rangle^{\prime}$ in Equation (41) is not invariant under the number operator $\hat{N}=\sum_{k} \hat{a}_{k}^{\dagger} \hat{a}_{k}$, while the Hamiltonian $\hat{\mathcal{H}}$ (Equation (28)) itself commutes with $\hat{N}$,

$$
e^{i \theta \hat{N}}|\boldsymbol{\alpha}\rangle^{\prime}=\left|e^{-i \theta} \boldsymbol{\alpha}\right\rangle^{\prime} ; \quad e^{i \theta \hat{N}} \hat{\mathcal{H}} e^{-i \theta \hat{N}}=\hat{\mathcal{H}} .
$$

Even though, the operator $e^{i \theta \hat{N}}$ applied to $|\boldsymbol{\alpha}\rangle^{\prime}$ produces a different state $\left|e^{-i \theta} \boldsymbol{\alpha}\right\rangle^{\prime}$, it leaves the scalar product invariant

$$
{ }^{\prime}\left\langle e^{-i \theta} \boldsymbol{\alpha} \mid e^{-i \theta} \boldsymbol{\alpha}\right\rangle^{\prime}={ }^{\prime}\langle\boldsymbol{\alpha} \mid \boldsymbol{\alpha}\rangle^{\prime} .
$$

This result immediately gives a clue that similar to harmonic oscillator coherent states, one can also multiply the fermionic coherent state by an arbitrary phase factor $e^{-i \theta}$ without changing any physical property. From the symmetry point of view, the situation is quite interesting for fermionic fields. So, we elaborate this issue a little more.

With the help of Equation (21), the overlap of the coherent states $|\boldsymbol{\alpha}\rangle^{\prime}$ defined by Equation (41) can be evaluated as follows

$$
{ }^{\prime}\left\langle\boldsymbol{\alpha}^{\prime} \mid \boldsymbol{\alpha}\right\rangle^{\prime}\left\langle\boldsymbol{\alpha} \mid \boldsymbol{\alpha}^{\prime}\right\rangle^{\prime}=\exp \left(-V \sum_{n}\left(\alpha_{n}^{* \prime}-\alpha_{n}^{*}\right)\left(\alpha_{n}^{\prime}-\alpha_{n}\right)\right) .
$$

Equation (46) implies that in the limit $V \rightarrow \infty$, any two coherent states $|\boldsymbol{\alpha}\rangle^{\prime},\left|\boldsymbol{\alpha}^{\prime}\right\rangle^{\prime}$ become orthogonal. So, the states with different phase factors, $|\boldsymbol{\alpha}\rangle^{\prime},\left|e^{-i \theta} \boldsymbol{\alpha}\right\rangle^{\prime}$ are macroscopically distinct. This novel findings of our analysis confirms the following points:

- Similar to BEC, the condensed state of FD gas [14], realized as a coherent state, forms a degenerate manifold parametrized by a phase variable $0<\theta<2 \pi$ [15].

- The microscopic Hamiltonian $(\hat{\mathcal{H}})$ (Equation (28)) has global U(1) symmetry, since it is invariant under multiplication by a constant phase factor to the wavefunction of the system, $\hat{\psi} \rightarrow e^{i \theta} \hat{\psi}$.

- The specific macroscopic ground condense state of Reference [14], viewed as a fermionic coherent state, does not possess such symmetry. The phase factor to the state $|\boldsymbol{\alpha}\rangle^{\prime}$ produces a different state 
altogether. This observation has a very fundamental consequence because the existence of BEC is always accompanied by spontaneous breaking of such symmetry $[87,88]$.

This enables us to establish BKC, in a similar spirit to BEC for bosons, as a condensation-like coherence among fermions [15].

\section{Discussion}

We elucidate an appealing feature of a largely uncharted territory of the condensation for the FD gas in close association with the Bose gas. The basic idea behind our present formulation is whether the condensed phase of the FD gas as a state of macroscopic coherence, can be described in terms of a fermionic coherent state. Such an approach gives an equivalent result to the problem of a fixed number of particles $N$ in the limit $N \longrightarrow \infty$ for BEC case [7-9]. We first summarize our main results of the present mini-review as follows:

- In Section 2, we present basic idea behind the Born-Green reciprocity principle [12,13,16-24,40-43], the assumption of its validity is the hypothesis of our work. The main conclusion of this section is that the highest momentum of a particle allowed by the reciprocity principle is proportional to that of the reduced rest mass of the particle in appropriate units.

- In Section 3, we discuss the Kothari-Nath approach [14,48] which assesses the statistical and thermodynamical properties of an assembly of noninteracting gas of fermions. We emphasize thereafter, unlike BEC, the most conspicuous feature of BKC: The condensation is primarily guided by the density of states rather than only statistics itself.

- In Section 4, we outline the coherence theory for fermionic fields following Cahill and Glauber [39]. Like quantum theory of optical coherence [77-80,89] describes the statistical properties and coherence of photons in quantum optics, we discuss the fermionic coherence theory can be shown to play a similar role for the description of fermionic atom-counting experiments $[64,74,75,85]$. Most important result of this section is the fermionic coherent state which is the eigenstate of the fermionic creation operators: An unitary displaced state of all filled up modes which arises due to the very special property of fermionic fields.

- In Section 5, our formulation portrays that the condensed phase of BKC can be recognized by the fermionic coherent state where the unitary displacement operator displaces in principle an infinitely dense filled up modes. This is similar in spirit to that of harmonic oscillator coherent states defined as unitarily displaced vacuum state which are used to represent BEC as a coherent matter wave. Our formulation combined with thermodynamic consideration allows us to characterize the condensate in terms of fermionic order parameter. It forms an essential ingredient for the demonstration of thermodynamic limit and spontaneous breaking of guage symmetry of the state comprising FD statistics. Most remarkably, similar to their bosonic counterpart, the coherent and the rotated coherent state can be distinguished as macroscopically distinct ground states of the FD gas. This enables us to capture the essence of BKC as "condensation-like coherence" among fermions, likewise BEC is treated as "coherent matter wave" for bosons .

Secondly, as mentioned earlier, an important point of discussion is to decipher whether $b$ is universal or not. From the standpoint of Equation (8), it appears that the constant $b$ is universal, since its value depends on classical electron radius $a$ and Planck's constant $\hbar$ which are the natural length and energy scales of all elementary particles. Presumably, one has

$$
a=\frac{e^{2}}{m_{0} c^{2}}=\frac{\hbar}{137 m_{0} c}=2.81 \times 10^{-13} \mathrm{~cm},
$$

where $m_{0}$ is the mass of the electron. Therefore [41]

$$
b=\hbar / a=137 m_{0} c .
$$


Moreover, one may define a primary absolute constant, namely the Born constant,

$$
\mathrm{B}=b / a=0.0085 \mathrm{gm} \mathrm{s}^{-1},
$$

originally postulated by M. Born [40] as the characteristic constant for the reciprocity principle in the same way as $c$ is the characteristic for relativity and $h$ for quantum theory.

However, the modern estimate for the electron radius $\left(10^{-20} \mathrm{~cm}\right)$ largely deviates from the "classical electron radius" [90] and thus poses a serious question on the "universality" of the Born constant $\mathrm{B}$ and the parameters in Born-Kothari (BK) model. By contrary, following reciprocity principle, BKC is associated with the maximum momentum $(\mu c)$ of a particle, i.e., the minimum wavelength predicted by its Compton's wavelength $\left(\lambda_{c}\right)$. It may not a priori clear whether the current electron radius indicates a modified $b$ value and therefore a modified Born constant $B$ or the highest momentum state in the BKC can be better determined by $\lambda_{c}$, independent of the values $a$ and $b$. Although, it is interesting to note when the classical electron radius is used [Confer Equation (47)], the two procedures give the same results for the electrons since $\lambda_{e}=137 a$. It is also worth noting that the reduced Compton's wavelength, $\lambda_{c}=\frac{\lambda}{2 \pi}=0.39 \times 10^{-10} \mathrm{~cm}$ and the simplest theoretical model due to Brodsky and Drell [91]

$$
\left|g_{e}-2\right|=R / \lambda_{c}
$$

with electron gyromagnetic ratio $\left|g_{e}-2\right|=1.1 \times 10^{-10} \mathrm{~cm}$, sets an upper bound on the electron radius, $R \approx 10^{-20} \mathrm{~cm}[92]$ and upon the rest energy of the particles out of which the electron is made. This results fits surprisingly well with the Large Electron-Positron Collider (LEP) accelerator data for electron energies $(\sim 200 \mathrm{GeV})$ which is five orders of magnitude large compared to the $0.5 \mathrm{MeV} / c^{2}$ rest mass of the electron. This indicates that the Compton's wavelength $\lambda_{c}$ could be a better candidate with respect to the parameters $a$ and $b$ for the estimation of the highest momentum for the condensate in BKC. However, if the Compton's wavelength is considered to be fundamental for the determination of the maximum momentum, one must admit the following problem:

- The energy scale at which the physical evidence of the modification in the density of states should appear in terms of Compton's wavelength for electrons (i.e., energy $\gg 1.41 \times 10^{8} \mathrm{eV}$, estimated by Born [12]), has been achieved decades ago [47]; therefore, one has to conclude that the estimation of the critical density in terms of the Compton's wavelength is far from realistic, and it was introduced into the discussion only for historical reasons.

- Present understanding in view of the last twenty years of experimental achievements on electrons energies reaching $\sim 200 \mathrm{GeV}$, implies that the effect of reciprocity principle could only be present at energies which are $\gtrsim 1 \mathrm{TeV}$.

- It seems therefore that the initial proposal for such scales introduced by Born [12] in his original theory of reciprocity, needs to be updated in the light of last 70 years of theory and experimental progress in high energy physics.

Finally, we stress that our approach, however, is not in contradiction to the standard pairing theory for fermions $[93,94]$ which merits attention in its own right, for the understanding of the behaviour of trapped atomic Fermi gases at ultracold temperatures [95-99], both as a degenerate quantum system [100] and as a possible precursor to a paired Fermi condensate [101-103]. It is also possible to account for a Bardeen-Cooper-Schrieffer (BCS)-like condensation incorporating Grassmann variables, which calls for a separate discussion [66-69]. Here, instead, we invoke the seldom used but reasonable basis for the possible existence of a condensed phase for the FD gas [14] that is based on the hypothesis of reciprocity principle $[12,13,17,21,40,41,43,104-106]$, which is considered one of the cornerstones for the development of the theory of elementary particles $[16,18,20,22-24,42]$ and other related fields [25,35-38]. As evident filling up a Fermi sea of real particles at an energy scale of $\mathrm{MeV}$ or even more is not only difficult but hardly imaginable. However, this should not lead 
to misunderstanding that the reciprocity principle is in contradiction with the known experimental results in atomic or condensed matter physics which are accessible via standard relativistic means. However, measuring a small effect at very high energy (due to the modified density of states) could be an excellent indirect proof for the experimental verification of the reciprocity principle itself.

We conclude our discussion with the following notes:

- In our previous paper [15], we have given only the theoretical formulation that accounts for the condensation of FD gas as close equivalent to BE gas. However, the nature of the highest momentum state where condensation takes place was not looked into. More precisely we did not specify what determines the value of the highest momentum for a given system of fermionic particles and what is its physical significance.

- In this mini-review we address all these issues in more detail. We hope our approach based on an unified analysis of different aspects of formulation of quantum theory and relativity may shed more light on the condensation phenomena of elementary fermions of astrophysical significance.

\section{Conclusions}

In summary, we stress that motivated by the Born's reciprocity theory, we present some interesting results based on a toy model of an ideal gas of non-interacting fermions. From this point of view, the present work may be valuable because even if the reciprocity theory does not hold, it would be possible that the level structure, for example, of certain condensed matter systems resemble to it. From the discussion of our previous section as well as experimental evidence on the possible validity of the Born's reciprocity theory, we conclude that

- If the reciprocity theory remains valid, its effects should be observable only at energy scales $\gtrsim \mathrm{TeV}$.

- In the BK model, a phenomenon like BKC could happen, and it could be modeled with our formalism based on coherent states with Grassmann variables.

- If a fermionic system presents a similar spectrum which is predicted by the BK theory, i.e., a high degeneracy around certain energy value inside a continuous band, then a similar phenomenon could appear only in the high density limit.

\section{Open Issues}

So far we have considered only non-interacting Fermi gas which is a good zeroth-order approximation for a dilute gas of fermions, where we neglect the effects of atom-atom interactions which are predominantly short-ranged and are therefore weak. For such dilute spin-polarized Fermi gases, the $s$-wave scattering amplitude vanishes due to the antisymmetric nature of the many fermionic wave function. The next leading order, $p$-wave scattering is small at low energy, hence one can safely ignore its effect and assume that the repulsive effect is mainly due to the Pauli exclusion principle rather than atom-atom interactions [107].

However, one can argue that at high energy when interaction terms are added, interacting fermions would simply invalidate the basic assumptions and the condensation-like phenomenon will not be relevant or even possible. In other ways, one could pose several different interesting questions: Can one obtain interacting, renormalisable, local or causal quantum field theories? If non-locality and or causality are lost can it be regained in some limits? We do not think a satisfactory answer to these questions can be given at this stage. So, we keep these issues as a list of future open problems.

Finally we anticipate the difficulty in achieving the BKC in an experiment with actual condensed matter systems $[15,108]$. Since it would correspond to fill up a Fermi sea of real particles at extremely high energy, one has to think whether one can create an analogous astrophysical conditions with fermionic atoms or molecules in the laboratory. We suppose that one needs the possible scaling relationship that may exist between different parameters, and then one may see how to mimic the gauge symmetry breaking in finite Fermi system with high number density. Recent experiments on high pressure superconductivity $[109,110]$ may throw new light in this direction. 
Acknowledgments: Thanks are due to the CSIR, Government of India, for partial financial support at the early stage of the research. Communication with Wolfgang Ketterle and his valuable comments on the Kothari-Nath paper and highest momentum value are thankfully acknowledged. The author is grateful to Deb Shankar Ray for critical reading of the manuscript and providing many useful advices. Nice inputs from Bimalendu Deb on probable practical implications and constant encouragement from Ramprasad Misra are sincerely acknowledged. We thank the anonymous referees for their several valuable suggestions and comments which definitely improved the quality of the paper.

Conflicts of Interest: The author declares no conflict of interest.

\section{Abbreviations}

The following abbreviations are used in this manuscript:

$\begin{array}{ll}\text { BEC } & \text { Bose-Einstein condensation } \\ \text { FD } & \text { Fermi-Dirac } \\ \text { BE } & \text { Bose-Einstein } \\ \text { BKC } & \text { Born-Kothari condensation } \\ \text { EM } & \text { Electromagnetic } \\ \text { BK } & \text { Born-Kothari }\end{array}$

\section{Appendix A. Rest Masses of Particles from Reciprocity Principle}

Particles with integral spin (or bosons), like photons and certain kinds of mesons, are generally supposed to have a wave function which satisfies a wave equation of the form [18]

$$
P \psi\left(x_{l}\right)=\kappa^{2} \psi\left(x_{l}\right), \quad P=p_{k} p^{k}, \quad p_{k}=i \frac{\partial}{\partial x^{k}},
$$

where the constant $\kappa$ is proportional to the rest mass of the particle. Going over to customary units one has

$$
\mu=(b / c) \kappa=(\hbar / a c) \kappa,
$$

as the actual rest mass of the observed particles. One can write the same wave function Equation (A1), also in the form

$$
F(P) \psi\left(x_{l}\right)=0,
$$

where $F(P)$ is any function of the form

$$
F(P)=F_{1}(P) P-\kappa^{2},
$$

and if $F_{1}(P)$ has no roots, Equation (A1) is the only solution. If, $F_{1}(P)$ is itself of the form $F_{2}(P)\left(P-\kappa^{2}\right)$ then the Equation (A1) will have solutions corresponding to particles of rest-mass $\mu_{1}=b \kappa_{1} / c$, also. Generalizing this, it can be seen quite easily that a wave equation of the type Equation (A1) may have solutions corresponding to particles with any number of different rest-masses [16].

This consideration can also be extended to particles with spin half by taking the argument of $F$ as $\eta$, instead of $P$ :

$$
\eta=\alpha^{k} p_{k}, \quad \alpha^{k} \alpha^{l}+\alpha^{l} \alpha^{k}=2 g^{k l}
$$


in terms of the standard notations of the metric tensor

$$
\begin{gathered}
g^{k l}=g_{k l}=0, \quad(k \neq l) ; \\
g_{11}=g_{22}=g_{33}=-1, \quad g_{44}=1 ; \\
g^{11}=g^{22}=g^{33}=-1, \quad g^{44}=1 ; \\
x^{k}=g^{k l} x_{l}, \quad x_{k}=g_{k l} x^{l} .
\end{gathered}
$$

In a similar way it can be shown to have solution satisfying equation

$$
F(\eta) \psi\left(x_{l}\right)=0,
$$

where $F(\eta)$ is any function of the form $\alpha^{k} p_{k}-\kappa$.

This way one obtains a vast generalization of wave mechanics describing any number of particles with different rest masses with the help of one single operator $F$, which is a function of the relativistic invariant $P$ or $\eta$, where $\eta^{2}=p_{k} p^{k}=P$. Supersymmetric generalizations of the reciprocity principle are of great interest in this regard $[34,44,45]$.

\section{Appendix B. Properties of the Displacement Operator}

The anti-commutivity of the Grassmann variables cancels on multiplication with that of fermionic operators. Thus, the operators $\hat{a}_{i}^{\dagger} \gamma_{i}$ and $\gamma_{j}^{*} \hat{a}_{j}$ commute for $i \neq j$. Hence, the displacement operator in Equation (19) may be written as the product

$$
\begin{aligned}
\hat{\mathcal{D}}(\gamma) & =\prod_{i} \exp \left(\hat{a}_{i}^{\dagger} \gamma_{i}-\gamma_{i}^{*} \hat{a}_{i}\right) \\
& =\prod_{i}\left[1+\hat{a}_{i}^{\dagger} \gamma_{i}-\gamma_{i}^{*} \hat{a}_{i}+\left(\hat{a}_{i}^{\dagger} \hat{a}_{i}-\frac{1}{2}\right) \gamma_{i}^{*} \gamma_{i}\right] .
\end{aligned}
$$

In the same logic, the annihilation operators $\hat{a}_{k}$ commutes with all the operators $\hat{a}_{i}^{\dagger} \gamma_{i}$ and $\gamma_{i}^{*} \hat{a}_{i}$ as long as $k \neq i$. Using Equation (A8), we may then compute the displaced annihilation operator by ignoring all modes except the $k$-th one:

$$
\begin{aligned}
\hat{\mathcal{D}}^{\dagger}(\gamma) \hat{a}_{k} \hat{\mathcal{D}}(\gamma) & =\prod_{i} \exp \left(\gamma_{i}^{*} \hat{a}_{i}-\hat{a}_{i}^{\dagger} \gamma_{i}\right) \cdot \hat{a}_{k} \cdot \prod_{j} \exp \left(\hat{a}_{j}^{\dagger} \gamma_{j}-\gamma_{j}^{*} \hat{a}_{j}\right) \\
& =\exp \left(\gamma_{k}^{*} \hat{a}_{k}-\hat{a}_{k}^{\dagger} \gamma_{k}\right) \cdot \hat{a}_{k} \cdot \exp \left(\hat{a}_{k}^{\dagger} \gamma_{k}-\gamma_{k}^{*} \hat{a}_{k}\right) \\
& =\hat{a}_{k}+\gamma_{k} .
\end{aligned}
$$

Similarly, we have

$$
\hat{\mathcal{D}}^{\dagger}(\gamma) \hat{a}_{k}^{\dagger} \hat{\mathcal{D}}(\gamma)=\hat{a}_{k}^{\dagger}+\gamma_{k}^{*}
$$

Using Equation (A9), one may verify the eigen value equation

$$
\begin{aligned}
\hat{a}_{k}|\gamma\rangle=\hat{a}_{k} \hat{\mathcal{D}}(\gamma)|0\rangle & =\hat{\mathcal{D}}(\gamma) \hat{\mathcal{D}}^{\dagger}(\gamma) \hat{a}_{k} \hat{\mathcal{D}}(\gamma)|0\rangle \\
& =\hat{\mathcal{D}}(\gamma)\left(\hat{a}_{k}+\gamma_{k}\right)|0\rangle \\
& =\gamma_{k} \hat{\mathcal{D}}(\gamma)|0\rangle=\gamma_{k}|\gamma\rangle
\end{aligned}
$$


while, Equation (26) can be verified by using Equation (A10) as

$$
\begin{aligned}
\hat{a}_{k}^{\dagger}|\boldsymbol{\alpha}\rangle^{\prime}=\hat{a}_{k}^{\dagger} \hat{\mathcal{D}}(\boldsymbol{\alpha})|\mathbf{1}\rangle & =\hat{\mathcal{D}}(\boldsymbol{\alpha}) \hat{\mathcal{D}}^{\dagger}(\boldsymbol{\alpha}) \hat{a}_{k}^{\dagger} \hat{\mathcal{D}}(\boldsymbol{\alpha})|\mathbf{1}\rangle \\
& =\hat{\mathcal{D}}(\boldsymbol{\alpha})\left(\hat{a}_{k}^{\dagger}+\alpha_{k}^{*}\right)|\mathbf{1}\rangle \\
& =\alpha_{k}^{*} \hat{\mathcal{D}}(\boldsymbol{\alpha})|\mathbf{1}\rangle=\alpha_{k}^{*}|\boldsymbol{\alpha}\rangle^{\prime}
\end{aligned}
$$

\section{References}

1. Einstein, A. Quantentheorie Des Einatomigen Idealen Gases; Wiley-VCH Verlag: Weinheim, Germany, 1925.

2. Bose, S.N. Plancks Gesetz und Lichtquantenhypothese. Zeitschrift für Physik 1924, 26, 178-181.

3. London, F. The lambda-Phenomenon of Liquid Helium and the Bose-Einstein Degeneracy. Nature 1938, 141, 643-644.

4. Anderson, M.H.; Ensher, J.R.; Matthews, M.R.; Wieman, C.E.; Cornell, E.A. Observation of Bose-Einstein Condensation in a Dilute Atomic Vapor. Science 1995, 269, 198-201,

5. Davis, K.B.; Mewes, M.O.; Andrews, M.R.; van Druten, N.J.; Durfee, D.S.; Kurn, D.M.; Ketterle, W. Bose-Einstein Condensation in a Gas of Sodium Atoms. Phys. Rev. Lett. 1995, 75, 3969-3973.

6. Bradley, C.C.; Sackett, C.A.; Tollett, J.J.; Hulet, R.G. Evidence of Bose-Einstein Condensation in an Atomic Gas with Attractive Interactions. Phys. Rev. Lett. 1995, 75, 1687-1690.

7. Leggett, A.J. Bose-Einstein condensation in the alkali gases: Some fundamental concepts. Rev. Mod. Phys. 2001, 73, 307-356.

8. Pethick, C.J.; Smith, H. Bose-Einstein Condensation in Dilute Gases; Cambridge University Press: Cambridge, UK, 2002.

9. Pitaevskii, L.P.; Stringari, S. Bose-Einstein Condensation; Clarendon Press: Oxford, UK, 2003.

10. Casadio, R.; Giugno, A.; Micu, O.; Orlandi, A. Thermal BEC Black Holes. Entropy 2015, 17, 6893-6924.

11. Watanabe, G.; Venkatesh, B.P.; Dasgupta, R. Nonlinear Phenomena of Ultracold Atomic Gases in Optical Lattices: Emergence of Novel Features in Extended States. Entropy 2016, 18, 118, doi:10.3390/e18040118.

12. Born, M. A Suggestion for Unifying Quantum Theory and Relativity. Proc. R. Soc. Lond. A Math. Phys. Eng. Sci. 1938, 165, 291-303.

13. Born, M. Relativity and quantum theory. Nature 1938, 141, 327-328.

14. Kothari, D.S.; Nath, B. 'Condensation' in Fermi-Dirac Statistics. Nature 1943, 151, 420-421.

15. Ghosh, A.; Ray, D.S. Born-Kothari condensation in an ideal Fermi gas. Phys. Rev. A 2017, 95, 022129, doi:10.1103/PhysRevA.95.022129.

16. Born, M.; Green, H.S.; Cheng, K.C.; Rodriguez, A.E. L.-Quantum Theory of Rest-Masses. Proc. R. Soc. Edinb. Sect. A Math. Phys. Sci. 1949, 62, 470-488.

17. Green, H.S. Quantized Field Theories and the Principle of Reciprocity. Nature 1949, 163, 208-209.

18. Born, M. Reciprocity Theory of Elementary Particles. Rev. Mod. Phys. 1949, 21, 463-473.

19. Wigner, E.P. Events, Laws of Nature, and Invariance Principles. Science 1964, 145, 995-999.

20. Yukawa, H. Quantum Theory of Non-Local Fields. Part I. Free Fields. Phys. Rev. 1950, 77, 219-226.

21. Born, M. Non-Localizable Fields and Reciprocity. Nature 1950, 165, 269-270.

22. Pais, A.; Uhlenbeck, G.E. On Field Theories with Non-Localized Action. Phys. Rev. 1950, 79, 145-165.

23. Yukawa, H. Structure and Mass Spectrum of Elementary Particles. II. Oscillator Model. Phys. Rev. 1953, 91, 416-417.

24. Born, M. The Born-Einstein Letters: Correspondence between Albert Einstein and Max and Hedwig Born from 1916 to 1955; Macmillan: New York, NY, USA, 1971.

25. Freidel, L.; Leigh, R.G.; Minic, D. Born reciprocity in string theory and the nature of spacetime. Phys. Lett. B 2014, 730, 302-306.

26. Low, S.G. Reciprocal Relativity of Noninertial Frames and the Quaplectic Group. Found. Phys. 2006, 36, 1036-1069.

27. Wigner, E. On Unitary Representations of the Inhomogeneous Lorentz Group. Ann. Math. 1939, 40, 149-204.

28. Bargmann, V.; Wigner, E.P. Group Theoretical Discussion of Relativistic Wave Equations. Proc. Natl. Acad. Sci. USA 1948, 34, 211-223.

29. Mackey, G.W. Induced Representations of Locally Compact Groups I. Ann. Math. 1952, 55, 101-139. 
30. Mackey, G.W. Induced Representations of Locally Compact Groups II. The Frobenius Reciprocity Theorem. Ann. Math. 1953, 58, 193-221.

31. Mackey, G.W. Infinite-dimensional group representations. Bull. Am. Math. Soc. 1963, 69, 628-686.

32. Freidel, L.; Leigh, R.G.; Minic, D. Quantum gravity, dynamical phase-space and string theory. Int. J. Mod. Phys. D 2014, 23, 1442006, doi:10.1142/S0218271814420061.

33. Freidel, L.; Leigh, R.G.; Minic, D. Modular Spacetime and Metastring Theory. J. Phys. Conf. Ser. 2017, 804, 012032, doi:10.1088/1742-6596/804/1/012032.

34. Gibbons, G.W.; Herdeiro, C.A.R. Born-Infeld theory and stringy causality. Phys. Rev. D 2001, 63, 064006, doi:10.1103/PhysRevD.63.064006.

35. Castro, C. On Born's deformed reciprocal complex gravitational theory and noncommutative gravity. Phys. Lett. B 2008, 668, 442-446.

36. Veneziano, G. A Stringy Nature Needs Just Two Constants. EPL Europhys. Lett. 1986, 2, doi:10.1209/0295-5075/2/3/006.

37. Prugovecki, E. Principles of Quantum General Relativity; World Scientific: Singapore, 1995.

38. Connes, A. Noncommutative Geometry; Academic Press: London, UK, 1994.

39. Cahill, K.E.; Glauber, R.J. Density operators for fermions. Phys. Rev. A 1999, 59, 1538-1555.

40. Born, M. Application of "Reciprocity" to Nuclei. Proc. R. Soc. Lond. A Math. Phys. Eng. Sci. 1938, 166, 552-557.

41. Born, M. Some remarks on reciprocity. Proc. Indian Acad. Sci. Sect. A 1938, 8, 309-314.

42. Born, M. Unification of the Theories of Photon and Meson. Nature 1944, 154, 764-765.

43. Born, M. Reciprocity Theory of Electrodynamics. Nature 1949, 164, 281-282.

44. Low, S.G. Canonically relativistic quantum mechanics: Representations of the unitary semidirect Heisenberg group, $\mathrm{U}(1,3) \otimes_{s} \mathrm{H}(1,3)$. J. Math. Phys. 1997, 38, 2197-2209.

45. Morgan, S. Supersymmetric Born Reciprocity. Ph.D. Thesis, University of Tasmania, Hobart, Australia, 2003.

46. Delbourgo, R.; Lashmar, D. Born Reciprocity and the 1/r Potential. Found. Phys. 2008, 38, 995-1010.

47. Jaeckel, J.; Spannowsky, M. Probing MeV to $90 \mathrm{GeV}$ axion-like particles with LEP and LHC. Phys. Lett. B 2016, 753, 482-487.

48. Nath, B. Thermodynamics of a Fermi-Dirac gas obeying Born's modified quantum statistics. Proc. Indian Acad. Sci. 1943, 9, 257-264.

49. Greiner, W. Quantum Mechanics: Special Chapters; Springer: Heidelberg, Germany, 1998.

50. Yang, C.N. Concept of Off-Diagonal Long-Range Order and the Quantum Phases of Liquid He and of Superconductors. Rev. Mod. Phys. 1962, 34, 694-704.

51. Schwinger, J. The Theory of Quantized Fields. IV. Phys. Rev. 1953, 92, 1283-1299.

52. Berezin, F.A. The Method of Second Quantization; Academic Press: New York, NY, USA, 1966.

53. Amelino-Camelia, G.; Freidel, L.; Kowalski-Glikman, J.; Smolin, L. Principle of relative locality. Phys. Rev. D 2011, 84, 084010, doi:10.1103/PhysRevD.84.084010.

54. Chu, C.S.; Furuta, K.; Inami, T. Locality, Causality and Noncommutative Geometry. Int. J. Mod. Phys. A 2006, 21, 67-82.

55. Amelino-Camelia, G.; Freidel, L.; Kowalski-Glikman, J.; Smolin, L. Relative locality: A deepening of the relativity principle. Gen. Relat. Gravit. 2011, 43, 2547-2553.

56. Brown, L. Quantum Field Theory; Cambridge University Press: Cambridge, UK, 1992.

57. Das, A. Field Theory: A Path Integral Approach; World Scientific: New Delhi, India, 2002.

58. Zinn-Justin, J. Quantum Field Theory and Critical Phenomena; Oxford University Press: New York, NY, USA, 1989.

59. Dalton, B.J.; Jeffers, J.; Barnett, S.M. Phase Space Methods for Degenerate Quantum Gases; Oxford University Press: New York, NY, USA, 2015.

60. Dalton, B.; Jeffers, J.; Barnett, S. Grassmann phase space methods for fermions. II. Field Theory. Ann. Phys. 2017, 377, 268-310.

61. Mrówczyński, S. Wigner functional of fermionic fields. Phys. Rev. D 2013, 87, 065026, doi:10.1103/PhysRevD.87.065026.

62. Corney, J.F.; Drummond, P.D. Gaussian Quantum Monte Carlo Methods for Fermions and Bosons. Phys. Rev. Lett. 2004, 93, 260401, doi:10.1103/PhysRevLett.93.260401.

63. Viennot, D.; Moro, O. Adiabatic transport of qubits around a black hole. Class. Quant. Gravity 2017, 34, 055005, doi:10.1088/1361-6382/aa5b5c. 
64. Braungardt, S.; Rodríguez, M.; Sen(De), A.; Sen, U.; Glauber, R.J.; Lewenstein, M. Counting of fermions and spins in strongly correlated systems in and out of thermal equilibrium. Phys. Rev. A 2011, 83, 013601, doi:10.1103/PhysRevA.83.013601.

65. Shen, H.Z.; Qin, M.; Xiu, X.M.; Yi, X.X. Exact non-Markovian master equation for a driven damped two-level system. Phys. Rev. A 2014, 89, 062113, doi:10.1103/PhysRevA.89.062113.

66. Efetov, K.B.; Pépin, C.; Meier, H. Exact Bosonization for an Interacting Fermi Gas in Arbitrary Dimensions. Phys. Rev. Lett. 2009, 103, 186403, doi:10.1103/PhysRevLett.103.186403.

67. Hu, H.; Jiang, L.; Liu, X.J.; Pu, H. Probing Anisotropic Superfluidity in Atomic Fermi Gases with Rashba Spin-Orbit Coupling. Phys. Rev. Lett. 2011, 107, 195304, doi:10.1103/PhysRevLett.107.195304.

68. Plimak, L.I.; Collett, M.J.; Olsen, M.K. Langevin equations for interacting fermions and Cooper-like pairing in trapped one-dimensional fermions. Phys. Rev. A 2001, 64, 063409, doi:10.1103/PhysRevA.64.063409.

69. Tempere, J.; Klimin, S.N.; Devreese, J.T. Effect of population imbalance on the Berezinskii-Kosterlitz-Thouless phase transition in a superfluid Fermi gas. Phys. Rev. A 2009, 79, 053637, doi:10.1103/PhysRevA.79.053637.

70. Ghosh, A.; Sinha, S.S.; Ray, D.S. Fermionic oscillator in a fermionic bath. Phys. Rev. E 2012, 86, 011138, doi:10.1103/PhysRevE.86.011138.

71. Shi, W.; Zhao, X.; Yu, T. Non-Markovian fermionic stochastic Schrodinger equation for open system dynamics. Phys. Rev. A 2013, 87, 052127, doi:10.1103/PhysRevA.87.052127.

72. Ghosh, A.; Sinha, S.S.; Ray, D.S. Canonical formulation of quantum dissipation and noise in a generalized spin bath. Phys. Rev. E 2012, 86, 011122, doi:10.1103/PhysRevE.86.011122.

73. Ho, S.H.; Li, W.; Lin, F.L.; Ning, B. Quantum decoherence with holography. J. High Energy Phys. 2014, 2014, 170, doi:10.1007/JHEP01(2014)170.

74. Ghosh, A. Parametric control in coupled fermionic oscillators. Phys. Rev. A 2014, 90, 043820, doi:10.1103/PhysRevA.90.043820.

75. Ghosh, A. Parametrically coupled fermionic oscillators: Correlation functions and phase-space description. Phys. Rev. A 2015, 91, 013835, doi:10.1103/PhysRevA.91.013835.

76. Tyc, T.; Hamilton, B.; Sanders, B.C.; Oliver, W.D. No Approximate Complex Fermion Coherent States. Found. Phys. 2007, 37, 1519-1539.

77. Glauber, R.J. The Quantum Theory of Optical Coherence. Phys. Rev. 1963, 130, 2529-2539.

78. Glauber, R.J. Coherent and Incoherent States of the Radiation Field. Phys. Rev. 1963, 131, 2766-2788.

79. Cahill, K.E.; Glauber, R.J. Ordered Expansions in Boson Amplitude Operators. Phys. Rev. 1969, 177, 1857-1881.

80. Cahill, K.E.; Glauber, R.J. Density Operators and Quasiprobability Distributions. Phys. Rev. 1969, 177, 1882-1902.

81. Barik, D.; Banerjee, D.; Ray, D.S. Quantum Brownian Motion in c-Numbers: Theory and Application; Nova-Science: New York, NY, USA, 2009.

82. Sinha, S.S.; Ghosh, A.; Ray, D.S. Quantum Smoluchowski equation for a spin bath. Phys. Rev. E 2011, 84, 031118, doi:10.1103/PhysRevE.84.031118.

83. Ghosh, A.; Sinha, S.S.; Ray, D.S. Quantum Dissipation in a spin bath; Application to Chemical Dynamics. Proc. Indian Acad. Sci. Sect. A 2014, 80, 949-998.

84. Sinha, S.S.; Ghosh, A.; Ray, D.S. Decay of a metastable state induced by a spin bath. Phys. Rev. E 2011, 84, 041113, doi:10.1103/PhysRevE.84.041113.

85. Glauber, R.J. Quantum Theory of Optical Coherence: Selected Papers and Lectures; Wiley-VCH: Weinheim, Germany, 2007.

86. Bogoliubov, N.N. On the theory of superfluidity. J. Phys. USSR 1947, 11, 23-32.

87. Yukalov, V.I. Bose-Einstein condensation and gauge symmetry breaking. Laser Phys. Lett. 2007, 4, $632-647$.

88. Yukalov, V.I. Basics of Bose-Einstein condensation. Phys. Part. Nucl. 2011, 42, 460-513.

89. Glauber, R.J. Quantum theory of coherence. In Quantum Optics; Kay, S.M., Maitland, A., Eds.; Academic Press: New York, NY, USA, 1970; Chapter 3, pp. 53-125.

90. Dehmelt, H. A Single Atomic Particle Forever Floating at Rest in Free Space: New Value for Electron Radius. Phys. Scr. 1988, 1988, 102-110.

91. Brodsky, S.J.; Drell, S.D. Anomalous magnetic moment and limits on fermion substructure. Phys. Rev. D 1980, 22, 2236-2243.

92. Dehmelt, H. Experiments with an isolated subatomic particle at rest. Rev. Mod. Phys. 1990, 62, 525-530. 
93. Leggett, A.J. Quantum Liquids: Bose Condensation and Cooper Pairing in Condensed-Matter Systems; Oxford University Press: Oxford, UK, 2006.

94. Leggett, A.J.; Zhang, S. The BEC-BCS Crossover: Some History and Some General Observations; Springer: Berlin, Germany, 2012; pp. 33-47.

95. Stoof, H.T.C.; Houbiers, M.; Sackett, C.A.; Hulet, R.G. Superfluidity of Spin-Polarized ${ }^{6}$ Li. Phys. Rev. Lett. 1996, 76, 10-13.

96. Giorgini, S.; Pitaevskii, L.P.; Stringari, S. Theory of ultracold atomic Fermi gases. Rev. Mod. Phys. 2008, 80, 1215-1274.

97. Gurarie, V.; Radzihovsky, L. Resonantly paired fermionic superfluids. Ann. Phys. 2007, 322, 2-119.

98. Greiner, M.; Regal, C.A.; Jin, D.S. Atomic Physics 19: XIX International Conference on Atomic Physics; American Institute of Physics: College Park, MA, USA, 2004.

99. Ketterle, W.; Inguscio, M.; Salomon, C. Proceedings of the Varenna "Enrico Fermi" Summer School; Societ Italiana di Fisica: Bologna, Italy, 2007.

100. DeMarco, B.; Jin, D.S. Onset of Fermi Degeneracy in a Trapped Atomic Gas. Science 1999, 285, $1703-1706$.

101. Regal, C.A.; Greiner, M.; Jin, D.S. Observation of Resonance Condensation of Fermionic Atom Pairs. Phys. Rev. Lett. 2004, 92, 040403; doi:10.1103/PhysRevLett.92.040403.

102. Zwierlein, M.W.; Stan, C.A.; Schunck, C.H.; Raupach, S.M.F.; Kerman, A.J.; Ketterle, W. Condensation of Pairs of Fermionic Atoms near a Feshbach Resonance. Phys. Rev. Lett. 2004, 92, 120403, doi:10.1103/PhysRevLett.92.120403.

103. Zwierlein, M.W.; Schunck, C.H.; Stan, C.A.; Raupach, S.M.F.; Ketterle, W. Formation Dynamics of a Fermion Pair Condensate. Phys. Rev. Lett. 2005, 94, 180401, doi:10.1103/PhysRevLett.94.180401.

104. Landé, A. Sommerfeld's Fine Structure Constant and Born's Reciprocity. Phys. Rev. 1939, 56, 482-483.

105. Landé, A. The Structure of Electric Particles and the Number 137. Phys. Rev. 1939, 56, 486.

106. Born, M. XXI.-Reciprocity and the Number 137. Part I. Proc. R. Soc. Edinb. 1940, 59, $219-223$.

107. Butts, D.A.; Rokhsar, D.S. Trapped Fermi gases. Phys. Rev. A 1997, 55, 4346-4350.

108. New Model to Unravel Mysteries of Elementary Particles. 2017. Available online: http:/ /www.natureasia. com/en/nindia/article/10.1038/nindia.2017.45 (accessed on 7 September 2017).

109. Drozdov, A.P.; Eremets, M.I.; Troyan, I.A.; Ksenofontov, V.; Shylin, S.I. Conventional superconductivity at 203 kelvin at high pressures in the sulfur hydride system. Nature 2015, 525, 73-76.

110. Mazin, I.I. Superconductivity: Extraordinarily conventional. Nature 2015, 525, 40-41.

(C) 2017 by the author. Licensee MDPI, Basel, Switzerland. This article is an open access article distributed under the terms and conditions of the Creative Commons Attribution (CC BY) license (http:/ / creativecommons.org/licenses/by/4.0/). 